\title{
Grifola gargal and G. sordulenta, Two Wild Edible Mushrooms with Perspectives as Biotech Products
}

\section{Pablo Daniel Postemsky}

Laboratory of Biotechnology of Edible and Medicinal Mushrooms, CERZOS (CONICET-UNS), Bahia Blanca, Argentina

*Corresponding author: Pablo D. Postemsky, Assistant Researcher, CERZOS-CONICET-UNS, Camino de la Carrindanga Km 7 , Bahía Blanca Argentina; Tel: +54 0291 4861666, ext-182; E-mail: pablop@criba.edu.ar

Received date: May 30, 2015; Accepted date: Dec 06, 2015; Published date: Dec 09, 2015

Copyright: (C) 2015 Pablo D. Postemsky, This is an open-access article distributed under the terms of the Creative Commons Attribution License, which permits unrestricted use, distribution, and reproduction in any medium, provided the original author and source are credited.

\section{Abstract}

Grifola gargal Singer and Grifola sordulenta Mont. (Singer) are two edible and medicinal mushroom species which occur in the AndinoPatagonian forests of Argentina and Chile. These woods that mainly consist of Nothofagus spp. are under increasing pressure with the consequent need of finding alternative sources for these mushrooms rather than gathering them. The main interest in these species is their culinary properties, which include a characteristic almond flavor present in the basidiomes, mycelium from submerged culture and solid-state fermented substrates, as well as the closely related Grifola frondosa, a well-known medicinal mushroom.

In recent years $G$. gargal and $G$. sordulenta have been studied for both their medicinal properties and culture conditions by researchers from Argentina, Chile, Japan and USA. However, neither industrial application nor commercial production has been undertaken. In this situation, a relevant role for fermentation technologies to develop possible future products from these wild-grown mushrooms is emphasized. Indeed, biotransformed wheat grains obtained through solid-state fermentation showed antioxidant and antigenotoxic activities which were comparable with those recorded from mycelium and basidiomes. The article comments on the up-to-date findings and state of research of these novel species, emphasizing some future research topics.

Keywords: Antioxidant; Mushrooms; Mycelium; Solid-state fermentation; Wheat grains

\section{Commentary}

The biodiversity of native Patagonian woods in both Argentina and Chile persists thanks to the existence of regulated protected areas and the efforts of teaching the values of forest diversity to local residents. There is concern about some endemic mushrooms that are declining as they are gathered for food and this type of impact is not well known and could potentially affect the ecological balance. Among them, Grifola gargal (known as "gargal or galgal") and G. sordulenta (no known common name) are two novel species belonging to the Polypore family that are related to Grifola frondosa (known as "maitake").

The latter is a well-known mushroom which possesses both demonstrated medicinal properties and biotechnological applications. Indeed, G. frondosa polysaccharides, phenolics and other molecules have been subjected to medicinal property studies and, certainly, some pharmaceutical products have been developed. Moreover, as a white rot fungus, its enzyme profile was evaluated for possible biotechnological applications.
The interest in both the edible and medicinal properties of $G$. gargal and $G$. sordulenta started in 1999, when researchers from Talca University (Chile) reported on the composition and biological activities of several herbs used by natives, including three species of mushrooms. Samples of $G$. gargal basidiomes were analyzed in respect to the nutritional content and biological activity [1]. This study showed a moderate effect in the DNA binding assay and also a strong hypotensive response in rats caused by $G$. gargal basidiomes collected in Valdivia (Chile).

By 2006, the first cultivation method approach was reported by Postemsky et al., [2]. An optimized method for agar culture and liquid culture was presented for both $G$. gargal and G. sordulenta. Starting with this report, a series of studies followed with the evaluation of different methods for obtaining $G$. gargal and $G$. sordulenta biomass through different culture systems at laboratory and pilot scales. These studies were supported by the Argentine Research Council and the Universidad Nacional del Sur (Argentina). The original specimens of these mushrooms were collected in the Lanín and Los Alerces National Parks (Argentina) for G. gargal and G. sordulenta, respectively.

A short time later, scientists from the University of Concepción and the National Institute of Agricultural Research (Chile) firstly described in detail the antioxidant activity of $G$. gargal basidiomes collected in Osorno (Chile), emphasizing the phenolics role in several in vitro assays activity [3,4]. More recently, an antioxidant activity report was presented by Postemsky and Curvetto [5], describing this attribute in mycelium extracts from both $G$. gargal and $G$. sordulenta obtained by different submerged culture methods and compared with liquid cultured mycelia from $G$. frondosa and basidiomes samples of $G$. gargal. This report illustrates how culture conditions can affect the antioxidant activity and phenolic content. Indeed, radical scavenging activity and the reduction power on ferrous ions were affected in different ways by either media components, the vessels used or the agitation speed. Auspiciously, some treatments showed a considerably higher activity than those that had previously been obtained with $G$. gargal basidiomes and $G$. frondosa mycelia. Also Harada et al. [6], recently reported the amelioration of atherosclerosis and the enhancement of regulatory T cells in a mouse model using $G$. gargal extracts of the basidiomes.

Development of protocols for the commercial production of $G$. gargal or G. sordulenta have not been achieved so far. However, some advances using artificial substrates and the classical methods for lignocellulolytic mushroom culture were reported by Shen and Royse [7] and Postemsky and Curvetto [5]. The constraint of basidiome supply may be a risk factor for natural populations due to the fact that the other alternative is to gather them from their habitats. Such activity can impose great danger to natural populations considering their low 
Citation: Pablo D. Postemsky (2015) Grifola gargal and G. sordulenta, Two Wild Edible Mushrooms with Perspectives as Biotech Products.

Page 2 of 2

abundance in the forest, where they mainly grow in relicts of the native Nothofagus tree species ( $N$. obliqua and $N$. dombeyi), although a certain ability to colonize other substrates was reported by Pozzi et al. [8], who described G. gargal colonizing the dead tissues of a poplar tree.

However, considering this scenario, it can be seen that these facts show that there is an opportunity for fermentation technologies to produce mushroom mycelium and molecules, using either submerged fermentation or solid-state fermentation. With this aim, Postemsky and Curvetto [9], produced a functional flour in a pilot-scale based on solid-state fermentation of wheat grains. Such biotransformed wheat grains presented the characteristic almond flavor, increasing the antioxidant capacity of wheat grains, but, was the beneficial biological activity found in a fermented substrate comparable to that obtained with mycelium or basidiomes? This hypothesis was shown to be true by performing an in vivo antigenotoxic assay using Drosophila melanogaster as the experimental model (Postemsky et al.) [10]. Fly larvae were cultured in a media containing the three above mentioned sources of $G$. gargal in combination with an anthropogenic carcinogen of anthracene nature. Moreover, a comparable effect of biotransformed wheat flour and mycelium from a submerged culture were found in $G$. sordulenta (unpublished results).

In addition, a recent report described the solid-state fermentation conditions of other substrates, including grains and sunflower seed hulls (Postemsky and Curvetto) [5]. In this study, it was described that almond aroma was present in any form of colonized substrate; moreover, it was found that sunflower seed hulls increased their protein content and laccase activity and also reduced the lignin fraction as a result of biodegradation. These analyses support further studies on the metabolic purification of interesting flavoring compounds, on pharmaceutical grade metabolites, such as ergothioneine, as was reported by Ito et al. [11], and also on enzymes for biotechnological purposes.

Future research on G. gargal and G. sordulenta may show if any of the different strains of these species, or even new ones (collected from noticeably different natural conditions), respond satisfactorily to artificial culture conditions. On the other hand, it must be evaluated whether different strains present distinctive biological activity, including the study of the effects of substrate composition and/or fermenting conditions on their metabolomic regulation. And, finally, research is necessary to compare the antitumoral and immunomodulatory activity of these species with the well-established medicinal mushroom $G$. frondosa.

\section{References}

1. Schmeda-Hirschmann G, Razmilic I, Gutierrez MI, Loyola JI (1999) Proximate composition and biological activity of food plants gathered by Chilean Amerindians. Economic Botany 53: 177-187.

2. Postemsky P, Matute G, Figlas D, Curvetto N (2006) Optimizing Grifola sordulenta and Grifola gargal growth in agar and liquid nutrient media. Micol Apl Int 18: 7-12.

3. Brujin J, Loyola C, Aqueveque P, Cañumir J, Cortéz M, France A (2008) Influence of heat treatment on the antioxidant properties of Grifola gargal hydro-alcoholic extracts. Micol Apl Int 20: 27-34.

4. Brujin J, Loyola C, Aqueveque P, Cañumir J, Cortéz M, France A (2009) Antioxidant properties of extracts obtained from Grifola gargal mushrooms. Micol Apl Int 21: 11-18.

5. Postemsky PD, Curvetto NR (2015) Solid-state fermentation of cereal grains and sunflower seed hulls by Grifola gargal and Grifola sordulenta. International Biodeterioration \& Biodegradation 100: 52-61.

6. Harada E, D'Alessandro-Gabazza CN, Toda M, Morizono T, ChelakkotGovindalayathil A, et. al (2014) Amelioration of Atherosclerosis by the New Medicinal Mushroom Grifola gargal Singer. International Biodeterioration \& Biodegradation 100: 52-61.

7. Shen Q, Royse DJ (2002) Effects of genotypes of maitake (Grifola frondosa) on biological efficiency, quality and crop cycle time. Applied Microbiology and Biotechnology 58: 178-182.

8. Pozzi C, Lorenzo L, Rajchenberg M (2009) Un hospedaje exótico del hongo comestible Grifola gargal (Basidiomycota, Fungi). Boletín la Soc Argent Botánica 44: 9-10.

9. Postemsky P, Curvetto N (2014) Enhancement of Wheat Grain Antioxidant Activity by Solid State Fermentation with Grifola spp. Journal of Medicinal Food 17: 543-549.

10. Postemsky PD, Palermo AM, Curvetto N (2011) Protective effects of new medicinal mushroom, Grifola gargal singer (higher Basidiomycetes), on induced DNA damage in somatic cells of Drosophila melanogaster. International Journal of Medicinal Mushrooms 13: 583-594.

11. Ito T, Kato M, Tsuchida H, Harada E, Niwa T, et al. (2011) Ergothioneine as an antioxidant /anti-inflammatory component in several edible mushrooms. Food Science and Technology Research 17: 103-110. 